\title{
Microbiological evaluation of infected wounds of the extremities in 214 adults
}

Laurent Holzapfel, Thierry Jacquet-Francillon, Jamil Rahmani, Pascal Achard, Eric Marcellin, Thierry Joffre, P Y Lallement, A Bousquet, Sabine Devaux, A Coupry

Centre Hospitalier de Bourge-en-Bresse, 01012 Bourg en Bresse, France

L Holzapfel

T Jacquet-Francillon

A Coupry

Centre Hospitalier, Levallois-Perret

J Rahmani

Centre Hospitalier, Annecy

P Achard

Hôpital Général, Orléans

E Marcellin

Centre Hospitalier, Vienne

T Joffre

Centre Hospitalier, Soissons

P Y Lallement

Centre Hospitalier, Saintes

A Bousquet

Centre Hospitalier, Argenteuil

$S$ Devaux

Correspondence and reprint requests to: Dr Holzapfel (e-mail:

lauholza@club-internet.fr).

Accepted 7 August 1998

\begin{abstract}
Objective-The aim of this multicentre prospective study was to analyse microbial pathogens cultured from an infected wound.

Methods-The study was performed in the emergency rooms of 10 public hospitals. All adult patients with a clinical diagnosis of cellulitis after a wound in the upper or lower extremities were included. Cultures were obtained with swabs from infected lesions. Micro-organisms cultured were identified by the usual methods and susceptibility testing was performed.

Results-The study population consisted of 214 patients, 153 men and 61 women, with a mean (SD) age of 40 (10) years. Wound cultures remained sterile in 28 cases and infected with micro-organisms in 186 cases. Of the 186 positive cultures, three were not identified. Of the 183 remaining cultures, one micro-organism was present in 132 patients $(62 \%)$ and several micro-organisms in 51 patients (24\%). A total of 248 micro-organisms were isolated in 183 patients. Staphylococcus and streptococcus were the most frequently isolated micro-organisms (56\% and $21 \%$ respectively) followed by Gram negative bacilli $(18 \%)$. Determination of the susceptibility to the antibiotics commonly used to treat wound infections showed resistance in some cases.

Conclusion-These results support the need always to take culture specimens from infected wounds for microbiological evaluation and antibiotic susceptibility determination, so that adapted chemotherapy can be prescribed.

(F Accid Emerg Med 1999;16:32-34)
\end{abstract}

Keywords: infected wounds; micro-organisms; cellulitis; antibiotics

Traumatic wound of the extremities is a common clinical problem and its treatment is well codified. Wound infection can be observed during the healing process. Serious infections such as anaerobic gangrenous cellulitis are infrequent and most cellulitis is caused by aerobic micro-organisms. The presentation is generally a localised infection, but serious sepsis can be seen. The consequences of cellulitis are to slow down scar formation and to cause aesthetic and functional sequelae.

Treatment of cellulitis consists of local care and intravenous or oral antibiotics. Microbial pathogens have been analysed in only a few studies with a small number of patients. ${ }^{1-3}$ Streptococcus pyogenes and Staphylococcus aureus were the most frequent micro-organisms found, but Gram negative bacilli were also isolated.

The aim of this multicentre prospective study was to analyse microbial pathogens cultured from an infected wound to better define microbiological sensitivities and the treatment that should be prescribed.

\section{Patients and methods}

STUDY POPULATION

The study was performed from October 1994 to June 1996 in the emergency rooms of 10 public non-teaching hospitals. All patients of 18 years old or more who came to the emergency room of the hospital with a clinical diagnosis of open cellulitis complicating a traumatic wound of the upper or lower extremities were included in the study. Open cutaneous cellulitis was defined as a localised process characterised by at least two of the following items: erythema, increased warmth, swelling, pain, and presence of purulent material. Patients were excluded for the following reasons: cellulitis of the head and neck, thorax and abdomen, history of antibiotic use in the preceding four days, surgical wound infection, illicit injection, likelihood of another diagnosis (for example, ulcers, impetigo, folliculitis, carbuncles, abscess, or other deep seated soft tissue infection, bites, erysipelas) or gangrenous cellulitis (cellulitis that has rapidly progressed with extensive necrosis of subcutaneous tissues and overlying skin). A standardised data collection form was used to collect the historical data. Informed consent was not required by the ethics committee of Lyon University.

\section{MICROBIOLOGICAL PROCEDURES}

Cultures were obtained with swabs from purulent material of cellulitis lesions without preparatory cleansing. Samples were placed in transport media and sent to the clinical laboratory, where they were plated onto blood, chocolate, and MacConkey agar. The agar plates were incubated for 48 hours at $35^{\circ} \mathrm{C}$ in $5 \%$ carbon dioxide. Micro-organisms cultured were identified by the usual methods and susceptibility testing was performed by the Bauer-Kirby disk method.

\section{Results}

\section{STUDY POPULATION}

The study population consisted of 214 patients, 153 men and 61 women, aged 18 to 92 
Table 1 Microbiological findings in 214 patients with positive cultures

\begin{tabular}{ll}
\hline Gram positive cocci & $\mathbf{1 9 6}(\mathbf{7 9 \% )}$ \\
Staphylococcus & $139(56 \%)$ \\
$\quad$ S aureus & 111 \\
S epidermidis & 8 \\
Other staphylococcus coagulase & 20 \\
$\quad$ negative & $51(21 \%)$ \\
Streptococcus & 30 \\
$\quad$ Group A & 5 \\
Group B & 16 \\
Other & $6(2 \%)$ \\
Enterococcus & $45(18 \%)$ \\
Gram negative bacilli & 4 \\
Acinetobacter & 11 \\
Enterobacter & 7 \\
Escherichia coli & 2 \\
Citrobacter & 2 \\
Klebsiella & 5 \\
Proteus & 1 \\
Providencia & 1 \\
Morganella & 4 \\
Aeromonas & 1 \\
Haemophilus & 5 \\
Pseudomonas aeruginosa & 2 \\
Pseudomonas sp & $2(1 \%)$ \\
Anaerobic bacteria & 1 \\
Peptostreptococcus & 1 \\
Propionibacterium & $5(2 \%)$ \\
Others & \\
\hline
\end{tabular}

Table 2 Susceptibility to antibiotics of isolated micro-organisms (\%)

\begin{tabular}{llll}
\hline & Staphylococcus & Streptococcus & $\begin{array}{l}\text { Gram } \\
\text { negative } \\
\text { bacilli }\end{array}$ \\
\hline Penicillin G & 18 & 94 & - \\
Amoxycillin (AMX) & 37 & 100 & 31 \\
$\begin{array}{l}\text { AMX-clavulanate } \\
\text { Oxacillin or }\end{array}$ & 99 & 100 & 51 \\
$\quad$ cloxacillin & 98 & 90 & - \\
$\begin{array}{l}\text { Gentamicin } \\
\text { Cefotaxime }\end{array}$ & 100 & - & 93 \\
Ticarcillin-clavulanate & - & 100 & 85 \\
\hline
\end{tabular}

years (mean (SD) 40 (10) years). Of the 214 patients, 133 had significant underlying conditions or an adverse lifestyle: smoking $(n=96)$, venous insufficiency $(\mathrm{n}=40)$, alcoholism $(n=25)$, diabetes mellitus $(n=10)$, drug addiction $(\mathrm{n}=4)$, and immunosuppressive treatment $(n=3)$. A hospital stay within the six preceding months was noted in 20 patients. Localisation of the infected wound was the upper extremities in 129 patients (60\%), lower extremities in 83 patients (39\%), and both in two patients. Length of the wound was $2.72 \mathrm{~cm}$ (range $0.3-15$ ). The wound was caused by a sharp object in 79 patients $(37 \%)$ and by a non-sharp object in 135 patients (63\%). Delay before infection was four days or less in 81 patients (39\%) and more than four days in 126 patients $(61 \%)$. Initial local wound care was performed in 111 patients and 43 of them were treated with oral antibiotics. No patient had a previous history of methicilin resistant $S$ aureus sensitivity or was known to have HIV.

MICROBIOLOGICAL FINDINGS

Of the 214 total wound cultures performed, 28 remained sterile and 186 grew microorganisms. Of the 186 positive cultures, three were not identified. Of the 183 remaining cultures, one micro-organism grew in 132 patients $(62 \%)$ and several micro-organisms in 51 patients $(24 \%)$. A total of 248 microorganisms were isolated in 183 patients. The micro-organisms are shown in table 1 ; table 2 shows their susceptibility to the antibiotics commonly used to treat cellulitis.

\section{Discussion}

Our results confirm the frequent occurrence of $S$ aureus and streptococcus in wound infections and the presence of Gram negative bacilli. Determination of the susceptibility to antibiotics showed frequent resistance to the antibiotics that are commonly used to treat wound infections.

This prospective study included an homogenous group of 214 adult patients with an infected wound of the extremities. Prior studies have included only a small number of patients with cellulitis of various body areas that were caused by different kinds of lesion (fungal infection, cutaneous ulceration, bites, illicit injections, erysipelas)..$^{124-8}$

In our study, cultures were obtained with swabs from purulent material of cellulitis lesions. In 1995 Uman and Kunin described the use of needle aspiration for diagnosis in patients with soft tissue infections. ${ }^{9}$ In different studies, the number of patients with positive cultures from needle aspiration were as follows: $3 / 30 \quad(10 \%),{ }^{6} 7 / 25 \quad(28 \%),{ }^{5} \quad 30 / 94 \quad(32 \%),{ }^{1}$ $20 / 42(48 \%),{ }^{7}$ and $21 / 31(68 \%){ }^{8}$ In most of these studies the authors concluded that needle aspiration may not be justified as a routine procedure for patients with cellulitis. Other microbiological sampling techniques have been used. In a study on 16 patients, incision and drainage yielded pus and cultures were positive in 10 cases. $^{2}$ In another study on 15 patients punch biopsy cultures were positive in 10 patients, aspirate and blood cultures were positive in five and two respectively, and cultures obtained with swabs from primary lesions in 10 patients showed the same organisms cultured by more invasive methods. ${ }^{4}$

Table 3 summarises microbiological data obtained in these studies. Comparison with our results is difficult because in these studies patients were not homogeneous, presented with

Table 3 Microbiological data in patients with cellulitis of the extremities

\begin{tabular}{|c|c|c|c|c|c|c|}
\hline Reference & No of patients & $\begin{array}{l}\text { No (\%) of patients } \\
\text { with positive culture }\end{array}$ & Staphylococcus (\%) & Streptococcus (\%) & $\begin{array}{l}\text { Gram negative } \\
\text { bacilli }(\%)\end{array}$ & Other micro-organisms (\%) \\
\hline Ginsberg $^{2} \ddagger$ & - & 12 & 43 & 57 & 0 & 0 \\
\hline Hook et $a l^{4} \delta$ & 50 & $13(26)$ & 45 & 36 & 9 & 9 \\
\hline \multicolumn{7}{|l|}{ Sigurdsson and } \\
\hline Gudmundsson ${ }^{1} \dagger$ & 94 & $30(32)$ & 56 & 24 & 15 & 5 \\
\hline Fleischer $e t a l^{\top} \dagger$ & 42 & $20(48)$ & 58 & 33 & 8 & 0 \\
\hline Santos $e t a l^{\beta} \dagger$ & 31 & $21(68)$ & 41 & 41 & 18 & 0 \\
\hline Our study` & 214 & $183(86)$ & 56 & 21 & 18 & 3 \\
\hline
\end{tabular}

Specimen collection: ${ }^{\star}$ swab; †needle aspiration; łneedle aspiration and incision culture; §swab, needle aspiration, and punch biopsy culture. 
cellulitis of various body areas that was caused by different kinds of lesion, and different sampling methods were used. Nevertheless our results are consistent with previous microbiological studies. Staphylococcus and streptococcus were the predominant micro-organisms (56\% and $21 \%$ respectively) and Gram negative bacilli were found in $18 \%$ of patients.

In our study patients with cellulitis due to anaerobic micro-organisms were not included, and microbiological techniques were not specially designed to isolate these micro-organisms because gangrenous cellulitis is very infrequent compared with aerobic cellulitis. Anaerobic micro-organisms were isolated in two cases. In a study in which all biopsy specimen homogenates were cultivated anaerobically, Clostridium perfringens and Clostridium paraperfringens were isolated in one patient in addition to $S$ aureus. ${ }^{4}$ In another study two patients had anaerobic streptococcal cellulitis, which was on the lower extremity in both cases. ${ }^{1}$

In the different studies analysing microorganisms cultivated from an infected wound, susceptibility of these micro-organisms to the different antibiotics routinely used to treat these infections were not reported. In our study, determination of the susceptibility to antibiotics showed frequent resistance to these commonly used antibiotics. Our results showed that presumptive antibiotherapy with penicillin $G$ or amoxycillin was not indicated. Amoxycillin-clavulanate, oxacillin, or cloxacillin could be used in staphylococcal and/or streptococcal infections but were not indicated in cases of Gram negative bacilli infections. Cefotaxime might be indicated, but Gram negative bacilli were sensitive in only $85 \%$ of cases. Ticarcillin-clavulanate and gentamicin could be used in most Gram negative infections. In many hospitals a prescription of amoxycillin-clavulanate is given while the culture results are awaited. ${ }^{10}$ Erythromycin is used for penicillin allergic patients. The present study shows that this approach is adequate in eight out of 10 cases.
In conclusion, this study confirms the frequent occurrence of $S$ aureus and streptococcus in wound infections and the presence of Gram negative bacilli. Determination of the susceptibility to antibiotics showed frequent resistance to the antibiotics commonly used to treat wound infections. These results support the need to systematically take culture specimens from an infected wound for microbiological evaluation and antibiotic susceptibility determination, so that an adapted chemotherapy can be prescribed. We suggest that such studies could be undertaken as a regular audit tool, in order to monitor antibiotic sensitivities.

Participating centres: Dr J Rahmani, Dr A M Canzi (LevalloisParticipating centres: Dr J Rahmani, Dr A M Canzi (LevalloisPerret), Dr P Achard, Dr A Sedaillan (Annecy), Dr E Marcellin, Dr E Borderon (Orleans), Dr T Joffre, Dr D Fevre (Vienne), Dr
P Y Lallement, Dr C Bourquigny-Saison (Soissons), Dr A $\mathrm{P}$ Y Lallement, Dr C Bourquigny-Saison (Soissons), Dr A
Bousquet, Dr T Porcher (Saintes), Dr T Jacquet-Francillon, Dr Bousquet, Dr T Porcher (Saintes), Dr T Jacquet-Francillon, Dr
A Coupry (Bourg en Bresse), Dr S Devaux, Dr F Le Turdu A Coupry (Bourg en Bresse), Dr S Devaux, Dr F Le Turdu
(Argenteuil), Dr J L Le Bivic, Dr P Mehl (Cognac), Dr J M (Argenteuil), Dr J L Le Bivic, Dr P Me

Coulaud, Dr A Mangeol (Montfermeil).
Presented to RICA (interdisciplinary meeting on antiPresented to RICA (interdisciplinary meeting on anti-
infectious chemotherapy), 5-6 December 1996, Paris, France. The study was sponsored by Smith Kline Beecham, Laboratoires Pharmaceutiques,

Nanterre, France. 1 Sigurdsson AF, Gudmundsson S. The etiology of bacterial cellulitis as determined by

2 Ginsberg MB. Cellulitis: analysis of 101 cases and review of the literature. South Med $\mathcal{F} 1981 ; 74: 530-3$

3 Benarbia S, Holzapfel L, Jacquet-Francillon T, et al. Etude microbiologique des plaies surinfectées chez les patients admis au service d'accueil des urgences (abstract). Réanimation Urgences 1994;3:115.

4 Hook EW, Hooton TM, Horton CA, et al. Microbiologic evaluation of cutaneous cellulitis in adults. Arch Intern Med 1986;146:295-7.

5 Sachs MK. The optimum use of needle aspiration in the bacteriologic diagnosis of cellulitis in adults. Arch Intern Med 1990;150:1907-12.

6 Newell P, Norden CW. Value of needle aspiration in bacteriologic diagnosis of cellulitis in adults. $\mathrm{f}$ Clin Microbiol 1988;26:401-4.

7 Fleisher G, Ludwig S, Campos J. Cellulitis: bacterial etiology, clinical features and laboratory findings. $\mathcal{F}$ Pediatr 1980;97:591-3.

8 Santos JI, Jacobson JA, Swensen P, et al. Cellulitis: treatment with cefoxitin compared with multiple antibiotic therapy. with cefoxitin compared with

9 Uman SJ, Kunin CM. Needle aspiration in the diagnosis of soft tissue infections. Arch Intern Med 1975;135:959-61.

10 Lammers RL. Principles of wound management. In Roberts JR, Hedges JR eds. Clinical procedures in emergency medicine. Philadephia: WB Saunders, 1985: 478-530. 